\title{
Review Article \\ Mechanisms of Omega-3 Polyunsaturated Fatty Acids in Prostate Cancer Prevention
}

\author{
Zhennan Gu, ${ }^{1,2}$ Janel Suburu, ${ }^{2}$ Haiqin Chen, ${ }^{1}$ and Yong Q. Chen ${ }^{1,2}$ \\ ${ }^{1}$ State Key Laboratory of Food Science and Technology, School of Food Science and Technology, Jiangnan University, \\ Wuxi 214122, China \\ ${ }^{2}$ Department of Cancer Biology, Wake Forest University School of Medicine, Medical Center Boulevard, Winston-Salem, \\ NC 27157, USA
}

Correspondence should be addressed to Yong Q. Chen; yqchen@wakehealth.edu

Received 6 March 2013; Revised 2 May 2013; Accepted 8 May 2013

Academic Editor: Gabriella Calviello

Copyright (c) 2013 Zhennan Gu et al. This is an open access article distributed under the Creative Commons Attribution License, which permits unrestricted use, distribution, and reproduction in any medium, provided the original work is properly cited.

This review focuses on several key areas where progress has been made recently to highlight the role of omega-3 polyunsaturated fatty acid in prostate cancer prevention.

\section{Introduction}

The health benefits of omega-3 polyunsaturated fatty acids (n-3 PUFA), mainly eicosapentaenoic acid (EPA 20:5) and docosahexaenoic acid (DHA, 22:6), have been long known. Epidemiologic studies dating back to the 1970s were among the first to suggest that dietary PUFA may be beneficial in preventing disease $[1,2]$. Still today, studies continue to demonstrate the health benefits of n-3 PUFA; however, the mechanisms of action of n-3 PUFA are still not fully understood. Many new discoveries have advanced our understanding about the activities of n-3 PUFA against human disease. For example, DHA-receptor GPR120 has been demonstrated to play a role in sensing and controlling obesity and metabolic syndrome [3]; the recently identified omega-3 mediators, resolvins, and protectins have been demonstrated to have anti-inflammatory and proresolving activities [4]. The purpose of this review is to highlight the recent advances in our understanding of the mechanisms by which n-3 PUFA modulate prostate cancer development.

\section{Fatty Acids}

There are two major classes of PUFA: n-6 and n-3. Unlike saturated and monounsaturated fatty acid, PUFA cannot be synthesized de novo by mammals because they lack the required enzymes and, therefore, PUFA must be obtained from the diet. The n- 6 and n-3 PUFA also cannot be interconverted in mammals, but within each series, their metabolism can produce various lipids that differ in chain length and number of double bonds. Linoleic acid (LA, 18:2n-6) is an n-6 PUFA found in high concentration in grains as well as many seeds and meats. LA serves as a substrate to be converted into a longer fatty acid, arachidonic acid (AA, 20:4 n-6), via a series of oxidative desaturation and elongation reactions. Of the $n-3$ fatty acids, alpha linolenic acid (ALA, 18:3n-3) is found at moderate levels in plants, seeds, leafy vegetables, legumes, and nuts. ALA is not metabolized efficiently to longer-chain n-3 PUFA, such as EPA and DHA.

Although they belong to two distinct families, n-3 and n-6 PUFA are metabolized by some of the same enzymes, specifically, delta-5-desaturase and delta-6-desaturase. Excess in one family of fatty acid can interfere with the metabolism of the other and alter their overall biological effects [5]. During n-6 PUFA conversion, delta-6-desaturase, or fatty acid desaturase 2 (FADS2), converts LA to gamma-linolenic acid (GLA, 18:3 n-6). This enzyme represents a rate-limiting step in the synthesis of AA from LA [6]. GLA is elongated to dihomogamma-linolenic acid (DGLA, 20:3 n-6) through a chain reaction of four enzymes: a condensation reaction of the fatty acyl chain with malonyl-CoA, catalyzed by an enzyme encoded by the ELOVL5 gene (elongation of very long-chain 
fatty acids, family member 5); a reduction reaction mediated by 3-ketoacyl-CoA reductase (KAR); a dehydration reaction catalyzed by 3-hydroxyacyl-CoA dehydratase (HACD); and a second reduction reaction catalyzed by trans-2,3-enoylCoA reductase (TECR). Finally, DGLA is converted to AA by delta-5 desaturase, or fatty acid desaturase 1 (FADS1) [6, 7]. Interestingly, malonyl-CoA, which is necessary for fatty acid elongation, is derived from the rate-limiting enzyme of the de novo fatty acid synthesis pathway, acetyl-CoA carboxylase. Fatty acid synthesis is well described as an overactive pathway in many cancers [8-11], and its upregulation may also contribute to the elongation of PUFA.

In contrast to AA, the efficiency of ALA conversion to DHA appears to be very low, below $5 \%$ in humans. Most ingested ALA is subject to beta-oxidation to provide energy, and only a small fraction is converted to EPA $[12,13]$. It was estimated that as low as $0.2 \%$ of ALA is converted to EPA, $64 \%$ of EPA to docosapentaenoic acid (DPA, 22:5 n-3), and $37 \%$ of DPA to DHA [14]. Thus, the overall amount of DPA and DHA converted from ALA is about $0.13 \%$ and $0.05 \%$ of the starting ALA, respectively. These findings suggest that any contributions from the fatty acid synthesis pathway toward PUFA metabolism most likely favor n-6, rather than n-3, PUFA elongation. It is also very likely that synthesis of the longer n-3 fatty acids from ALA within the body is competitively hindered by the n-6 analogues. It has been reported that the $n-3$ conversion efficiency is greater in women, possibly because of the importance of meeting the DHA demands of the fetus and neonate [14].

\section{PUFA and Cancer}

Total fat intake and the ratio of n- 6 to $n-3$ PUFA in the Western diet have increased significantly since the Industrial Revolution $[15,16]$. Increased fat consumption has been associated with the development of specific types of cancer such as breast, colon, and pancreatic and prostate cancers, with the notable exception of n-3 PUFA, which show protective effects against colon, breast, and prostate cancers in a number of experimental systems [17-23]. Epidemiological studies about the association of dietary fat and cancer suggests a protective effect of n-3 PUFA and a promoting effect of n-6 PUFA on cancer. Most clinical data regarding the effects of dietary fat on cancers are observational [24], and the results of such studies are mixed, as many fail to demonstrate a significant association between n-3 PUFA and reduced prostate cancer risk or tumor growth [20, 25-27].

The Western diet contains disproportionally high amounts of n-6 PUFA and low amounts of n-3 PUFA, denoted as a high n- 6 to n-3 PUFA ratio. Most data regarding the effects of high dietary n-6 PUFA are positively associated with prostate cancer incidence [28-30]. In a study of Jamaican men undergoing prostate biopsy for elevated PSA levels, a positive correlation was observed between n-6 fatty acid LA and Gleason score and n-6 (LA) to n-3 (DHA) ratio in erythrocyte membranes and prostate tumor volume [31]. By comparing PUFA content from malignant and benign prostatic tissues from the same prostate specimens, a Swedish research group found that n-6 PUFA and n-6 PUFA precursors were significantly higher in malignant tissues. This finding further demonstrates that n-6 dietary fat is associated with prostate carcinogenesis [28]. In racespecific analyses based on a case-control study comprising 79 prostate cancer cases and 187 controls, Williams and colleagues found that a high ratio of n-6 to n-3 fatty acids may increase the overall risk of prostate cancer among white men and possibly increase the risk of high-grade prostate cancer among all men [29].

At the same time, epidemiological literature on the association of n-3 PUFA and cancer, including correlational studies and migrational studies, suggest a protective role played by $n-3$ PUFA. In a recent population-based prospective cohort study of 90,296 Japanese subjects, Sawada et al. reported that consumption of n-3-rich fish or n-3 PUFA, particularly EPA, DPA, and DHA, appears to protect against the development of hepatocellular carcinoma (HCC) [32]. In another population-based prospective study in Japan, there was an inverse relationship between marine n-3 PUFA intake and the risk of colorectal cancer, but this association was only statistically significant in the proximal site of the large bowel [33]. Chavarro et al. performed a nested case-control study by analyzing blood samples of 14,916 healthy men and concluded that higher blood levels of long-chain n-3 fatty acids were associated with a reduced risk of prostate cancer [34]. Szymanski et al. conducted a meta-analysis of fish intake and prostate cancer by focusing on the incidence of prostate cancer and prostate cancer-specific mortality. Their results did not establish a protective association of fish consumption with prostate cancer incidence but showed a significant $63 \%$ reduction in prostate cancer-specific mortality [35].

The results of correlational studies are mixed, some of them failing to demonstrate a statistically significant effect. Several confounding factors could account for the inconsistent results on the association between n-3 PUFA and prostate cancer. First, population-based studies mainly rely on data from self-reported dietary fatty acid intake or from estimates based on national consumption, and these assessments correlate poorly with direct measurements of fatty acids in patient samples. In addition, the actual intake in n-3 PUFA may be too low for a protective effect in some cases. Second, the ratio of n- 6 to $n-3$ fatty acids may be more important than the absolute amount of n-3 PUFA, as suggested by animal and human studies [16, 36]. Using a prostate-specific Pten knockout mouse prostate cancer model, we showed that a ratio of n- 6 to n-3 below 5 was effective in slowing cancer progression [3]. Brown et al. reported that AA might potentiate the risk of metastatic prostate cancer cell migration and seeding at the secondary site in vivo, and lowering the $n-6 / n-3$ ratio in diet by uptake of n-3 PUFA might reduce this risk [37].

\section{Mechanisms of Action}

4.1. Integration of PUFAs into Plasma Membrane Glycerophospholipids. Although fatty acids are consumed at high levels in a typical Western diet, tumor cells display a strong 
dependence on de novo fatty acid synthesis $[9,10]$. The increased proliferation and metabolism of cancer cells could be the trigger for the abnormal requirement for fatty acid compared to normal cells. Most newly synthesized fatty acids are used to support membrane biogenesis in the form of glycerophospholipids, a class of lipids that are a major component of all cell membranes. Glycerophospholipids, including phosphatidylcholine (PC), phosphatidylserine (PS), phosphatidylethanolamine (PE), and phosphatidylinositol (PI), contain a diglyceride, a phosphate group, and a simple organic molecule, such as choline or serine.

Dietary PUFA can influence the fatty acid composition of glycerophospholipids in cell membranes. In mammals, the $s n-1$ position on the glycerol backbone of glycerophospholipids is usually linked to a saturated fatty acid such as stearic acid (SA, 18:0), and the $s n-2$ position is linked to an n-6 PUFA, such as AA. Feeding cells in culture, or animals, with n-3 PUFA can replace n- 6 with n- 3 fatty acid at the $s n-2$ position of glycerophospholipids; this is considered a diet-induced $s n$ 2 fatty acid moiety change $[17,38,39]$. We have analyzed the incorporation efficiency of PUFAs into glycerophospholipids in prostate cancer cells. Approximately $25 \%$ of input albuminconjugated fatty acids were incorporated into cells within 48 hours. The majority of these newly integrated PUFAs were in the form of PC and PE [40]. These data clearly suggest that the fatty acid at the $s n-2$ position of glycerophospholipids is influenced by cellular PUFA uptake.

n-3 PUFA influences cell membrane conformation and signaling dynamics. The greater density of n-3 PUFA, compared to n-6 PUFA, dictates their aggregation near the lipidwater interface. This characteristic can significantly affect plasma membrane properties, including membrane fluidity, phase behavior, and permeability [41]. These membrane perturbations can bring about changes associated with receptor activation, such as diffusional coupling of various peripheral proteins required in $\mathrm{G}$ protein-coupled receptor signaling (GPCR) $[42,43]$. Additionally, because of its high level of unsaturation, DHA has very poor affinity for cholesterol, which is enriched in lipid rafts of the cell membrane. Lipid rafts are important membrane domains for cell signaling as many receptors and proteins are enriched in this domain, such as epidermal growth factor receptor (EGFR) [44]. Hence, incorporation of n-3 PUFA into membrane lipids can disturb the formation of lipid rafts and suppress raftassociated cell signal transduction $[13,44]$.

The serine/threonine protein kinase AKT (protein kinase B) is activated in many solid tumors and hematological malignancies. AKT acts downstream of phosphatidylinositol 3-kinase (PI3K) signaling and is a key regulator of multiple survival pathways. AKT is known to phosphorylate and inactivate the proapoptotic Bcl-2 family member BAD as one of its prosurvival tactics [45]. Phosphatidylinositol (PI) is a negatively charged constituent of lipid membranes. Specific kinases phosphorylate the hydroxyl groups on positions $3^{\prime}$, $4^{\prime}$, or $5^{\prime}$ of the inositol ring, and PI3K primarily generates $\mathrm{PI}$-3,4,5-trisphosphate $\left(\mathrm{PIP}_{3}\right)$ from PI-4,5-bisphosphate $\left(\mathrm{PI}(4,5) \mathrm{P}_{2}\right) \cdot \mathrm{PIP}_{3}$ acts as a second messenger to activate pleckstrin homology $(\mathrm{PH})$ domain-containing proteins, including AKT. Conversely, $\mathrm{PIP}_{3}$ is hydrolyzed to $\mathrm{PI}(4,5) \mathrm{P}_{2}$ by PTEN, opposing the action of PI3K $[46,47]$. AKT can also be phosphorylated and activated by phosphoinositide-dependent kinase-1 (PDPK1), a PH domain-containing kinase downstream of PI3K (for review, see Franke, 2008) [48]. Hence, intracellular $\mathrm{PIP}_{3}$ plays a pivotal role in this $\mathrm{PI} 3 \mathrm{~K} / \mathrm{PIP}_{3} / \mathrm{AkT}$ cascade pathway.

Using prostate-specific Pten knockout mice, an immunecompetent, orthotopic prostate cancer model, and diets with defined PUFA levels, we found that n-3 fatty acid reduced prostate tumor growth, slowed histopathological progression, and increased survival, whereas n-6 fatty acid had opposite effects. Introducing an n-3 desaturase, which converts n-6 to n-3 fatty acid, into the Pten knockout mice fed an n-6 diet reduced tumor growth similarly to mice fed the n-3 diet. Tumors from mice on the n-3 diet had lower proportions of phosphorylated BAD and higher apoptotic indexes compared with tumors from mice on the n- 6 diet. These data suggest that n-3 PUFA can promote BAD-dependent apoptosis to modulate prostate cancer development [17]. We also found that PUFAs modify glycerophospholipid content. DHA can replace the fatty acid at the $s n-2$ position of the glycerol backbone, thereby changing the species of phospholipid. DHA also inhibited $\mathrm{AKT}^{\mathrm{T} 308}$ but not $\mathrm{AKT}^{\mathrm{S} 473}$ phosphorylation, altered $\mathrm{PIP}_{3}$ and phospho- $\mathrm{AKT}^{\mathrm{S} 473}$ protein localization, decreased $\mathrm{pPDPK}{ }^{\mathrm{S} 241}$-AKT and AKT-BAD interaction, and suppressed prostate tumor growth. Knockdown of BAD eliminated n-3 PUFA-induced cell death, and reintroduction of BAD restored the sensitivity to $\mathrm{n}-3$ fatty acids in vitro. Knockout of BAD diminished the suppressive effect of $n-3$ PUFA on prostate tumor growth in vivo. These data suggest that modulation of prostate cancer development by PUFA is mediated in part through the PI3K/AKT survival pathway $[17,40]$.

$\mathrm{Hu}$ et al. reported that n-3 PUFA-induced apoptosis in human prostate cancer cells occurs through upregulation of syndecan-1 (SDC-1) expression followed by concomitant suppression of PDPK1/AKT/BAD phosphorylation [49]. n3 fatty acids may also decrease cell proliferation and induce apoptotic cell death in human cancer cells by decreasing signal transduction through the $\mathrm{AKT} / \mathrm{NF} \kappa \mathrm{B}$ cell survival pathway and by modulating the PI3K/AKT/p38 MAPK pathway $[50,51]$.

4.2. PUFA Mediator. A common fate of unsaturated lipids released from the membrane is oxidation. AA is the precursor of highly bioactive lipid mediators metabolized by a number of enzymes belonging to the cyclooxygenase (COX) and lipoxygenase (LOX) families, as well as cytochrome P450. COXs have two well-characterized isoforms, COX1 and COX2. COX1 is a constitutively expressed gene in most tissues, whereas COX2 is an immediate-early response gene, which is strongly induced in many human malignancies [52]. Signal transduction of n-6 PUFA-derived lipids and the effect of these lipid mediators on the organism have been well characterized. For example, AA-derived lipid mediators are associated with a variety of activities, including inflammation and cancer. Evidence from human studies also supports the important role of COXs and LOXs in PUFA metabolism and 
cancer [53-56]. Because of its high expression in inflammation and cancer, COX2 has been the subject of intense study and proposed as a target for cancer therapy [57-59].

In contrast to n-6 PUFA, the metabolism of n-3 PUFA is not well understood. Interest in n-3 PUFA-derived lipid mediators began with observations of Greenland Eskimos whose diet is rich in marine-derived fish and showed lower mortality from coronary heart disease and lower prevalence of inflammation-related diseases, such as psoriasis, inflammatory bowel disease, asthma, rheumatoid arthritis, and other autoimmune diseases $[60,61]$. Serhan et al. reported that inflammatory exudates in the murine air pouch from mice treated with n-3 PUFA and aspirin contained a series of bioactive compounds. Using an unbiased lipidomics approach, they identified and named the EPA-derived Eresolvins (RvE1 and RvE2), DHA-derived D-resolvins (RvD1 and RvD2), and (neuro-)protectin (PD1) [62].

RvE1 and RvE2 are protective in a wide variety of disease models, mainly through their anti-inflammatory activities. RvE1 can resolve inflammation caused by bacterial infection of periodontal disease in a rabbit model [63], prevent neovascularization after oxygen-induced retinopathy [64], and suppress neutrophil infiltration in an acute peritonitis model [65]. RvD1, RvD2, and PD1 also have protective activities in a variety of animal models, including models of lung injury, insulin resistance, peritonitis, wound healing, and atherosclerosis [66]. RvD1 was shown to reduce leukocyte infiltration in murine inflammatory exudates [67] and RvD2 was shown to reverse inflammatory pain in mice [68]. PD1 has been reported to regulate amyloid beta secretion and thereby improve neuronal survival in a mouse model of Alzheimer's disease [69]. Although ample data indicate that these n-3 PUFA-derived mediators can resolve inflammation, little is known about their role in inflammation-related cancers, such as prostate and colon cancers.

Due to the existence of multiple oxygenases, the role of each enzyme in the development of prostate cancer has not been studied systematically in a single system or animal model. Furthermore, studies performed in animals rarely take diet into account. To systematically assess the interaction between oxygenases and dietary PUFA in a single animal model of prostate cancer, we knocked out Cox1, Cox2, Lox5, Lox12, or Lox15 in prostate-specific Pten null mice. Our preliminary results indicate that tumor growth was significantly increased in $\mathrm{Coxl}^{-/-}$Pten null mice on $\mathrm{n}-3$ diet compared to Coxl-wild-type Pten null littermates. This result suggests that Coxl is required for the protective effects of n-3 PUFA. Interestingly, tumor growth was decreased in n-6 PUFA fed Coxl ${ }^{-/-}$Pten-null mice compared to n-6 fed Coxlwildtype Pten-null mice, suggesting that n- 6 metabolites of Cox1 promote tumor growth. Loss of Cox 2 reduced prostate tumor growth on both n-3 and n-6 diets, suggesting that the suppressive effect of n-3 PUFA is independent of Cox2 metabolism. Loss of Lox 5 reduced prostate tumor growth on n-6 diet but had no effect on n-3 diet; loss of Lox12 or Lox15 did not affect prostate tumor growth on either diet. These results suggest that the promotion of prostate tumor growth by $n-6$ diet is dependent on Lox- 5 metabolism and both Lox12 and Lox15 metabolites are not critical for prostate cancer growth in this model (Chen et al., unpublished).

4.3. Fatty Acids Receptors. Lipids are ligands for cell-surface $G$ protein-coupled receptors (GPCRs), toll-like receptors (TLRs), and peroxisome proliferator-activated receptors (PPARs). G protein-coupled receptors (GPCRs) are important signaling molecules for many aspects of cellular function. They are members of a large family that share common structural motifs, such as seven transmembrane helices and the ability to activate heterotrimeric G proteins. Recently, several groups reported that unbound free fatty acids can activate GPCRs, including GPR40, GPR41, GPR43, GPR84, and GPR120 [3]. Short-chain fatty acids are specific ligands for GPR41 and GPR43, medium-chain fatty acids for GPR84, and long-chain fatty acids for GPR40 and GPR120 [70-73]. Activation of GPR84 receptor by medium-chain fatty acids triggered the production of the proinflammatory cytokines from leukocytes and macrophages. The function of GPR84 may be associated with chronic low-grade inflammationassociated disease [74].

GPR40 and GPR120 have been reported to be activated by long-chain fatty acids such as DHA, EPA, and AA [73, 75]. As a $G$ protein-coupled receptor, GPR40 can activate the phospholipase $\mathrm{C}$ and phosphatidylinositol signaling pathways [76]. Although GPR40 is preferentially expressed in pancreatic $\beta$-cells and is known to mediate insulin secretion [77], several groups showed that it is expressed in the brain where it mediates the antinociceptive activity of DHA $[78,79]$. Recently, Oh and others reported that GPR120 functions as an n-3 PUFA receptor in vitro and in vivo [3] and suggested that diminished activation of GPR120 can be an important contributor to obesity, insulin resistance, and tissue inflammation [80, 81]. GPR120 is highly expressed in adipose tissue, proinflammatory bone marrow-derived $\mathrm{CD}_{11 C^{+}}$macrophages (BMDCs), mature adipocytes, and monocytic RAW 264.7 macrophage cells. DHA strongly inhibited LPS-induced phosphorylation of JNK and IKK $\beta$, $\mathrm{I} \kappa \mathrm{B}$ degradation, cytokine secretion- and inflammatory gene expression level in GPR120-positive cells. These effects of DHA were completely prevented by GPR120 knockdown, demonstrating that these anti-inflammatory effects were specifically exerted through GPR120. An n-3 PUFA diet containing $27 \%$ fish oil led to improved insulin sensitivity with increased glucose infusion rates, enhanced muscle insulin sensitivity, and greater hepatic insulin sensitivity. The n-3 PUFA diet had no effect in the GPR120 knockout (KO) mice. On chow diets, the GPR120 KO mice showed moderate insulin resistance with no changes in food intake or body weight. On high-fat diet (HFD), the GPR120 KO mice gained more weight than wild-type controls [3]. In humans, GPR120 expression in adipose tissue is significantly higher in obese individuals than in lean controls [80]. Ichimura and colleagues compared sequences of GPR120 exons in obese populations and discovered a deleterious nonsynonymous mutation $(\mathrm{R} 270 \mathrm{H})$ [80]. Their population study showed that the GPR $120^{\mathrm{R} 270 \mathrm{H}}$ variant correlated with obesity. Further investigation in vitro demonstrated that the GPR $120^{\mathrm{R} 270 \mathrm{H}}$ 
variant was unable to respond to long-chain fatty acid stimulation. This inactive mutant of GPR120 may contribute to its significant association with obesity [80].

Toll-like receptors (TLRs) are transmembrane glycoprotein receptors that are important regulators of the innate immune system. TLRs are considered a link between innate (nonspecific) and adaptive (specific) immunity and contribute to the immune system's capacity to efficiently combat pathogens [82]. TLR expression is increased in tumors, including breast, colorectal, melanoma, lung, prostate, pancreatic, and liver cancer [83]. Activation of TLRs triggers a signaling cascade producing inflammatory cytokines that recruit components of the adaptive immune system to kill the pathogen [84]. Among the family of TLRs, TLR4 and TLR9 have been reported to be associated with prostate cancer [85-87]. Panigrahy et al. reported that saturated fatty acids activated, and DHA inhibited, TLR2- and TLR4-mediated proinflammatory activity in a cell culture system [88]. Saturated fatty acids may stimulate the TLR4 signaling pathway to trigger the production of proinflammatory mediators, which may contribute to neuronal death [89].

PPARs (PPAR $\alpha, \operatorname{PPAR} \beta / \delta$, and $\operatorname{PPAR} \gamma)$ are a superfamily of ligand-activated transcription factors and nuclear hormone receptors. The syndecan family of cell surface proteoglycans share a structure of small, conserved cytoplasmic and transmembrane domains and larger, distinct ectodomain. They are implicated in a variety of physiologic and pathologic processes such as nutrient metabolism, energy homeostasis, inflammation, and cancer. Growing evidence has demonstrated that PPAR $\gamma$ serves as a tumor suppressor in cancer (see review of Robbins and Nie, 2012) [90]. n-3 PUFA can induce apoptosis in human prostate cancer cells by activating the nuclear receptor PPAR $\gamma$ and upregulating the PPAR $\gamma$ target gene, syndecan-1 (SDC-1) [49, 91]. It has been suggested that n-3 PUFA induces cell apoptosis in prostate cancer via a mechanism of LOX15-mediated SDC1-dependent suppression of PDPK1/AKT/BAD phosphorylation $[49,91]$. SDC-1 upregulation by DHA has also been demonstrated in human breast cancer cells $[19,92-94]$ and in n-3 PUFA-enriched mammary glands and liver of fat-1 mice [95].

SDC-1 plays several important cellular functions. It regulates many steps of leukocyte recruitment in noninfectious inflammatory diseases, attenuates inflammation by modulating heparin sulfate-binding proinflammatory factors, and plays a key role in the normal remodeling of injured cardiac tissues [96]. Loss of cell surface SDC-1, seen in many carcinomas such as skin cancer and colorectal adenocarcinomas, favors acquisition of the metastatic phenotype in cancer cells [97]. There is very limited information about SDC-1 expression in prostate cancer. Some studies reported an inverse relationship between SDC-1 and Gleason score $[98,99]$, but a tissue microarray analysis in another study showed an increase in SDC-1 with tumor progression [100]. Our own studies have shown reduced expression of SDC1 in prostate cancer cell lines compared to normal prostate epithelial cells and lower expression in androgen-dependent LNCaP cells compared to androgen-independent PC3 and DU145 cells [49]. In a mouse prostate cancer model, the reduction in tumor growth as a result of dietary n-3 PUFA is accompanied by an increase in the expression of secreted SDC-1 $[49,101]$.

Several other receptors have been suggested as targets for n-3 PUFA action. Turk et al. reported that DHA can induce the alteration in both the lateral and subcellular localization of EGFR and suppress EGFR signaling, which suggests implications for the molecular basis of cancer prevention by DHA $[44,102]$. It is also reported that DHA can increase CD95 (Fas ligand death receptor) cell surface expression and may mediate CD95-induced apoptosis [103]. For more information about PUFA receptor interaction, please refer to a review by Lee et al. [104].

4.4. Other Mechanisms. Nuclear factor erythroid-2-related factor $2(\mathrm{Nrf} 2)$ is a basic leucine zipper transcription factor. $\mathrm{Nrf} 2$ is sequestered in the cytoplasm by Kelch-like ECHassociated protein 1 (Keap1) under basal conditions. When the cell is challenged by oxidative stress, Nrf2 is released from Keap1 inhibition, translocates to the nucleus, forms a complex with other factors, and activates transcription of genes containing an antioxidant response element (ARE) in their promoter region. Nrf2 has been reported to play an important role in lung injury reversal, human endothelial cell survival, neuroinflammation, hyperoxia, lung damage from cigarette smoking, and impaired function of macrophages [105]. Other studies suggest that Nrf2 suppresses inflammation by inhibiting $\mathrm{NF} \kappa \mathrm{B}$ activation through regulation of redox balance, calcium signaling, and PPARs [106]. Various human cancers, such as lung cancer, frequently exhibit increased levels of Nrf2 [107]. Downregulation of nuclear Nrf2 gene expression by RNAi-mediated silencing in nonsmall cell lung cancer inhibits tumor growth and increases efficacy of chemotherapy [108]. Cancer cells are suspected to hijack the Keap1-Nrf2 system as a means to acquire malignant properties. Indeed, the prognosis of patients carrying Nrf2-positve cancers is poor [105]. Oxidized n-3 fatty acids can react directly with the negative regulator of $\mathrm{Nrf2}$, Keap1, by dissociating them and inducing Nrf2-directed gene expression [109]. For example, n-3 PUFA mediators can activate Nrf2 in vascular endothelial cells to prevent oxidative stress-induced cytotoxicity [110]. DHA and EPA can induce Nrf2 expression and suppress lipopolysaccharide-(LPS-) induced inflammation [111]. Evidence of Nrf2-mediated response modulated by n-3 PUFA in prostate cancer came from a randomized clinical trial. Eighty-four men with low-risk prostate cancer were stratified based on self-reported dietary consumption of fish oil. Exploratory pathway analyses of rank-ordered genes revealed the modulation of Nrf2 or Nrf2-mediated oxidative response after 3 months of fish oil supplementation $(P=0.01)$ [112].

Calcium $\left(\mathrm{Ca}^{2+}\right)$ signaling is a ubiquitous mechanism in the control of cell function. The transient receptor potential channels (TRP channels) are 6 transmembrane-spanning proteins with both amino and carboxyl tails located on the intracellular side of the membrane. $\mathrm{Ca}^{2+}$ flux through TRP channels located in the plasma membrane and in the membranes of excitable intracellular organelles can promote changes in intracellular free $\mathrm{Ca}^{2+}$ concentrations and the 
TABLE 1: Mechanism of n-3 PUFA action.

\begin{tabular}{|c|c|c|}
\hline Target & Mechanism of action & References \\
\hline Membrane PIPs & Suppress PI3K/AKT/BAD survival pathway & {$[17,40]$} \\
\hline SDC-1 & Suppress $\mathrm{PI} 3 \mathrm{~K} / \mathrm{AKT} / \mathrm{BAD}$ and $\mathrm{AKT} / \mathrm{NF} \kappa \mathrm{B}$ survival pathway & [49] \\
\hline Unknown & Suppress AKT/NFאB or PI3K/AKT/p38 MAPK pathway & {$[50,51]$} \\
\hline GPR40 & Mediate insulin secretion, cell proliferation & [75-77] \\
\hline GPR120 & Anti-inflammation & {$[3,80]$} \\
\hline TLRs & Suppress the production of proinflammatory mediators & {$[88,89]$} \\
\hline PPARg & Induce cell apoptosis & {$[90]$} \\
\hline Nrf2 & Suppress inflammation, reduce oxidative stress & {$[106,109,110]$} \\
\hline Calcium signaling & Inhibit cell proliferation & {$[114]$} \\
\hline COXs & Promote inflammation & {$[52]$} \\
\hline LOXs & Promote inflammation & {$[53]$} \\
\hline E-resolvins & Resolve inflammation & {$[62]$} \\
\hline D-resolvins & Resolve inflammation & {$[62]$} \\
\hline
\end{tabular}

membrane potential, which can modulate the driving force for other ions and $\mathrm{Ca}^{2+}$ itself [113]. Evidence suggests that TRP channel function can be modulated both directly and indirectly by n-3 fatty acids [114]. It was demonstrated that DHA and EPA at physiological concentrations have the ability to evoke small currents, which seems to be dependent on the previous sensitization of the channel by protein kinase $\mathrm{C}$ (PKC). Whether these effects are due to the action of these PUFAs on the agonist binding site or are due to conformational changes caused by TRP protein interactions with the lipid bilayer requires further investigation [115]. Recent findings also indicate that TRP channel function can be modulated by $\mathrm{D}$ and $\mathrm{E}$ resolvins. Resolvin binding on GPCRs seems to be part of the mechanism underlying the resolvin-mediated regulation of TRP channel function [116]. DHA significantly reduces oxidative stress-induced endothelial cell $\mathrm{Ca}^{2+}$ influx. This effect might be associated, at least in part, with altered lipid composition in membrane caveolar rafts [117]. $\mathrm{Ca}^{2+}$ has been shown to be essential for increased cell proliferation in prostate cells [118]. Sun et al. observed significantly higher $\mathrm{Ca}^{2+}$ influx in prostate cancer cells. They reported that high ratio of $\mathrm{Ca}^{2+} / \mathrm{Mg}^{2+}$ facilitated $\mathrm{Ca}^{2+}$ influx and led to a significant increase in cell proliferation of prostate cancer [119]. Thus, one could speculate that n-3 PUFA might indirectly modulate prostate cancer growth by directly modulating $\mathrm{Ca}^{2+}$ influx.

\section{Conclusions}

Cancer incidence and mortality are high in the Western world and a high n-6 to n-3 PUFA ratio in the Western diet may be a contributing factor. There is much evidence to suggest that n-3 PUFA has antiproliferative effects in cancer cell lines, animal models, and humans. Direct effects on cancer cells and indirect effects on the host immune system (antiinflammation) likely contribute to the inhibitory effect of n-3 fatty acids on tumor growth; however, further investigation is warranted. n-3 PUFA may also regulate other complex metabolic processes, including $\beta$-oxidation, lipid release from glycerophospholipids, cellular signaling of membrane bound proteins, eicosanoid synthesis, and direct activation of nuclear receptors and gene transcription, all of which may influence the development and progression of prostate cancer. Overall, there seems to be an exceptionally broad potential for the mechanisms mediating cancer prevention by n-3 PUFA (summarized in Table 1). We expect that new research in lipidomics and metabolomics will provide new techniques and approaches to answering the many questions that remain regarding the mechanisms underlying the health benefits of n-3 PUFA.

\section{Abbreviations}

PUFA: Polyunsaturated fatty acid

EPA: Eicosapentaenoic acid (20:5, n-3)

DHA: Docosahexaenoic acid (22:6, n-3)

LA: $\quad$ Linoleic acid (18:2, n-6)

AA: $\quad$ Arachidonic acid (20:4, n-6)

ALA: Alpha linolenic acid (18:3, n-3)

FADS: Fatty acid desaturase

GLA: Gamma-linolenic acid (18:3, n-6)

DGLA: Dihomo-gamma-linolenic acid (20:3, n-6)

KAR: 3-Ketoacyl-CoA reductase

HACD: 3-Hydroxyacyl-CoA dehydratase

TECR: Trans-2,3-enoyl-CoA reductase

DPA: Docosapentaenoic acid (22:5, n-3)

PC: Phosphatidylcholine

PS: Phosphatidylserine

PE: $\quad$ Phosphatidylethanolamine

PI: Phosphatidylinositol

SA: $\quad$ Stearic acid (18:0)

GPCR: G Protein-coupled receptor

EGFR: Epidermal growth factor receptor

AKT: Serine/threonine protein kinase (protein kinase B)

PI3K: Phosphatidylinositol-3-kinase

$\mathrm{PIP}_{3}$ : PI-3,4,5-trisphosphate 
PH: Pleckstrin homology

PDPK1: Phosphoinositide-dependent kinase-1

SDC-1: Syndecan-1

COX: Cyclooxygenase

LOX: Lipoxygenase

RvE1: E-Resolvin1

RvD1: D-Resolvin1

TLR: Toll-like receptor

PPAR: Peroxisome proliferator-activated receptor

MCFA: Medium-chain fatty acid

BMDC: Bone marrow-derived $\mathrm{CD}_{11 C^{+}}$macrophage

KO: Knockout

HFD: High-fat diet

Nrf2: Nuclear factor erythroid-2-related factor 2

Keap1: Kelch-like ECH-associated protein 1

ARE: Antioxidant response element

LPS: Lipopolysaccharide

PKC: $\quad$ Protein kinase C.

\section{References}

[1] H. O. Bang, J. Dyerberg, and A. B. Nielsen, "Plasma lipid and lipoprotein pattern in Greenlandic West-coast Eskimos," The Lancet, vol. 1, no. 7710, pp. 1143-1145, 1971.

[2] J. Dyerberg, H. O. Bang, and N. Hjorne, "Fatty acid composition of the plasma lipids in Greenland Eskimos," The American Journal of Clinical Nutrition, vol. 28, no. 9, pp. 958-966, 1975.

[3] D. Y. Oh, S. Talukdar, E. J. Bae et al., "GPR120 is an Omega3 fatty acid receptor mediating potent anti-inflammatory and insulin-sensitizing effects," Cell, vol. 142, no. 5, pp. 687-698, 2010.

[4] A. Ariel and C. N. Serhan, "Resolvins and protectins in the termination program of acute inflammation," Trends in Immunology, vol. 28, no. 4, pp. 176-183, 2007.

[5] C. A. Daley, A. Abbott, P. S. Doyle, G. A. Nader, and S. Larson, "A review of fatty acid profiles and antioxidant content in grassfed and grain-fed beef," Nutrition Journal, vol. 9, no. 1, article 10, 2010.

[6] J. T. Bernert Jr. and H. Sprecher, "Studies to determine the role rates of chain elongation and desaturation play in regulating the unsaturated fatty acid composition of rat liver lipids," Biochimica et Biophysica Acta, vol. 398, no. 3, pp. 354-363, 1975.

[7] I. M. Berquin, I. J. Edwards, S. J. Kridel, and Y. Q. Chen, "Polyunsaturated fatty acid metabolism in prostate cancer," Cancer and Metastasis Reviews, vol. 30, no. 3-4, pp. 295-309, 2011.

[8] J. V. Swinnen, K. Brusselmans, and G. Verhoeven, "Increased lipogenesis in cancer cells: new players, novel targets," Current Opinion in Clinical Nutrition and Metabolic Care, vol. 9, no. 4, pp. 358-365, 2006.

[9] F. P. Kuhajda, "Fatty acid synthase and cancer: new application of an old pathway," Cancer Research, vol. 66, no. 12, pp. 59775980, 2006 .

[10] J. A. Menendez and R. Lupu, "Fatty acid synthase and the lipogenic phenotype in cancer pathogenesis," Nature Reviews Cancer, vol. 7, no. 10, pp. 763-777, 2007.

[11] J. Suburu and Y. Q. Chen, "Lipids and prostate cancer," Prostaglandins and Other Lipid Mediators, vol. 98, pp. 1-10, 2012.

[12] G. C. Burdge and P. C. Calder, "Conversion of $\alpha$-linolenic acid to longer-chain polyunsaturated fatty acids in human adults,"
Reproduction Nutrition Development, vol. 45, no. 5, pp. 581-597, 2005.

[13] C. M. Williams and G. Burdge, "Long-chain n-3 PUFA: plant v. marine sources," Proceedings of the Nutrition Society, vol. 65, no. 1, pp. 42-50, 2006.

[14] R. J. Pawlosky, J. R. Hibbeln, J. A. Novotny, and N. Salem, “Physiological compartmental analysis of $\alpha$-linolenic acid metabolism in adult humans," Journal of Lipid Research, vol. 42, no. 8, pp. 1257-1265, 2001.

[15] A. P. Simopoulos, "Evolutionary aspects of omega-3 fatty acids in the food supply," Prostaglandins Leukotrienes and Essential Fatty Acids, vol. 60, no. 5-6, pp. 421-429, 1999.

[16] A. P. Simopoulos, "The importance of the ratio of omega6/omega-3 essential fatty acids," Biomedicine and Pharmacotherapy, vol. 56, no. 8, pp. 365-379, 2002.

[17] I. M. Berquin, Y. Min, R. Wu et al., "Modulation of prostate cancer genetic risk by omega-3 and omega- 6 fatty acids," Journal of Clinical Investigation, vol. 117, no. 7, pp. 1866-1875, 2007.

[18] G. Calviello, F. Resci, S. Serini et al., "Docosahexaenoic acid induces proteasome-dependent degradation of $\beta$-catenin, down-regulation of survivin and apoptosis in human colorectal cancer cells not expressing COX-2," Carcinogenesis, vol. 28, no. 6, pp. 1202-1209, 2007.

[19] H. Sun, I. M. Berquin, R. T. Owens, J. T. O'Flaherty, and I. J. Edwards, "Peroxisome proliferator-activated receptor $\gamma$ mediated up-regulation of syndecan-1 by n-3 fatty acids promotes apoptosis of human breast cancer cells," Cancer Research, vol. 68, no. 8, pp. 2912-2919, 2008.

[20] I. M. Berquin, I. J. Edwards, and Y. Q. Chen, "Multi-targeted therapy of cancer by omega-3 fatty acids," Cancer Letters, vol. 269, no. 2, pp. 363-377, 2008.

[21] Y. Q. Chen, I. J. Edwards, S. J. Kridel, T. Thornburg, and I. M. Berquin, "Dietary fat-gene interactions in cancer," Cancer Metastasis Reviews, vol. 26, pp. 535-551, 2007.

[22] S. Wang, J. Wu, J. Suburu, Z. Gu, J. Cai et al., "Effect of dietary polyunsaturated fatty acids on castration-resistant Pten-null prostate cancer," Carcinogenesis, vol. 33, pp. 404-412, 2012.

[23] K. K. Carroll, "Dietary fat and cancer: specific action or caloric effect?” Journal of Nutrition, vol. 116, no. 6, pp. 1130-1132, 1986.

[24] M. Gerber, "Omega-3 fatty acids and cancers: a systematic update review of epidemiological studies," British Journal of Nutrition, vol. 107, Supplement 2, pp. 228-239, 2012.

[25] P. D. Terry, T. E. Rohan, and A. Wolk, "Intakes of fish and marine fatty acids and the risks of cancers of the breast and prostate and of other hormone-related cancers: a review of the epidemiologic evidence," The American Journal of Clinical Nutrition, vol. 77, no. 3, pp. 532-543, 2003.

[26] C. H. MacLean, S. J. Newberry, W. A. Mojica et al., "Effects of omega-3 fatty acids on cancer risk: a systematic review," Journal of the American Medical Association, vol. 295, no. 4, pp. 403-415, 2006.

[27] Y. Q. Chen, I. M. Berquin, L. W. Daniel et al., "Omega-3 fatty acids and cancer risk," Journal of the American Medical Association, vol. 296, no. 3, pp. 278-282, 2006.

[28] M. C. Schumacher, B. Laven, F. Petersson et al., "A comparative study of tissue $\omega-6$ and $\omega-3$ polyunsaturated fatty acids (PUFA) in benign and malignant pathologic stage $\mathrm{pT} 2$ a radical prostatectomy specimens," Urologic Oncology, vol. 31, no. 3, pp. 318-324, 2013.

[29] C. D. Williams, B. M. Whitley, C. Hoyo, D. J. Grant, J. D. Iraggi et al., "A high ratio of dietary n-6/n-3 polyunsaturated fatty acids 
is associated with increased risk of prostate cancer," Nutrition Research, vol. 31, no. 1, pp. 1-8, 2011.

[30] M. D. Brown, C. Hart, E. Gazi, P. Gardner, N. Lockyer, and N. Clarke, "Influence of omega-6 PUFA arachidonic acid and bone marrow adipocytes on metastatic spread from prostate cancer," British Journal of Cancer, vol. 102, no. 2, pp. 403-413, 2010.

[31] C. R. Ritch, R. L. Wan, L. B. Stephens et al., "Dietary fatty acids correlate with prostate cancer biopsy grade and volume in Jamaican men," Journal of Urology, vol. 177, no. 1, pp. 97-101, 2007.

[32] N. Sawada, M. Inoue, M. Iwasaki, S. Sasazuki, T. Shimazu et al., "Consumption of n-3 fatty acids and fish reduces risk of hepatocellular carcinoma," Gastroenterology, vol. 142, pp. 14681475, 2012.

[33] S. Sasazuki, M. Inoue, M. Iwasaki et al., "Intake of n-3 and n-6 polyunsaturated fatty acids and development of colorectal cancer by subsite: Japan Public Health Center-based prospective study," International Journal of Cancer, vol. 129, no. 7, pp. 17181729, 2011.

[34] J. E. Chavarro, M. J. Stampfer, H. Li, H. Campos, T. Kurth, and J. Ma, "A prospective study of polyunsaturated fatty acid levels in blood and prostate cancer risk," Cancer Epidemiology Biomarkers and Prevention, vol. 16, no. 7, pp. 1364-1370, 2007.

[35] K. M. Szymanski, D. C. Wheeler, and L. A. Mucci, "Fish consumption and prostate cancer risk: a review and metaanalysis," The American Journal of Clinical Nutrition, vol. 92, no. 5, pp. 1223-1233, 2010.

[36] N. Kobayashi, R. J. Barnard, S. M. Henning et al., "Effect of altering dietary $\omega-6 / \omega-3$ fatty acid ratios on prostate cancer membrane composition, cyclooxygenase- 2 , and prostaglandin E 2," Clinical Cancer Research, vol. 12, no. 15, pp. 4662-4670, 2006.

[37] M. D. Brown, C. A. Hart, E. Gazi, S. Bagley, and N. W. Clarke, "Promotion of prostatic metastatic migration towards human bone marrow stoma by Omega 6 and its inhibition by Omega 3 PUFAs," British Journal of Cancer, vol. 94, no. 6, pp. 842-853, 2006.

[38] M. C. Chabot, J. D. Schmitt, B. C. Bullock, and R. L. Wykle, "Reacylation of platelet activating factor with eicosapentaenoic acid in fish-oil-enriched monkey neutrophils," Biochimica et Biophysica Acta, vol. 922, no. 2, pp. 214-220, 1987.

[39] M. Picq, P. Chen, M. Perez, M. Michaud, E. Vericel et al., "DHA metabolism: targeting the brain and lipoxygenation," Molecular Neurobiology, vol. 42, pp. 48-51, 2010.

[40] Z. Gu, J. Wu, S. Wang et al., "Polyunsaturated fatty acids affect the localization and signaling of PIP3/AKT in prostate cancer cells," Carcinogenesis, 2013.

[41] S. R. Wassall and W. Stillwell, "Docosahexaenoic acid domains: the ultimate non-raft membrane domain," Chemistry and Physics of Lipids, vol. 153, no. 1, pp. 57-63, 2008.

[42] N. V. Eldho, S. E. Feller, S. Tristram-Nagle, I. V. Polozov, and K. Gawrisch, "Polyunsaturated docosahexaenoic vs docosapentaenoic acid - Differences in lipid matrix properties from the loss of one double bond," Journal of the American Chemical Society, vol. 125, no. 21, pp. 6409-6421, 2003.

[43] D. C. Mitchell, S. L. Niu, and B. J. Litman, "Quantifying the differential effects of DHA and DPA on the early events in visual signal transduction," Chemistry and Physics of Lipids, vol. 165, pp. 393-400, 2012.

[44] H. F. Turk, R. Barhoumi, and R. S. Chapkin, "Alteration of EGFR spatiotemporal dynamics suppresses signal transduction," PLoS One, vol. 7, no. 6, Article ID e39682, 2012.
[45] S. R. Datta, H. Dudek, T. Xu et al., "Akt phosphorylation of BAD couples survival signals to the cell- intrinsic death machinery," Cell, vol. 91, no. 2, pp. 231-241, 1997.

[46] T. Maehama and J. E. Dixon, "The tumor suppressor, PTEN/MMAC1, dephosphorylates the lipid second messenger, phosphatidylinositol 3,4,5-trisphosphate," Journal of Biological Chemistry, vol. 273, no. 22, pp. 13375-13378, 1998.

[47] J. A. Engelman, J. Luo, and L. C. Cantley, "The evolution of phosphatidylinositol 3-kinases as regulators of growth and metabolism," Nature Reviews Genetics, vol. 7, no. 8, pp. 606-619, 2006.

[48] T. F. Franke, "PI3K/Akt: getting it right matters," Oncogene, vol. 27, no. 50, pp. 6473-6488, 2008.

[49] Y. Hu, H. Sun, R. T. Owens et al., "Syndecan-1-dependent suppression of PDK1/Akt/Bad signaling by docosahexaenoic acid induces apoptosis in prostate cancer," Neoplasia, vol. 12, no. 10, pp. 826-836, 2010.

[50] P. D. Schley, H. B. Jijon, L. E. Robinson, and C. J. Field, "Mechanisms of omega-3 fatty acid-induced growth inhibition in MDA-MB-231 human breast cancer cells," Breast Cancer Research and Treatment, vol. 92, no. 2, pp. 187-195, 2005.

[51] J. L. D. Toit-Kohn, L. Louw, and A. M. Engelbrecht, "Docosahexaenoic acid induces apoptosis in colorectal carcinoma cells by modulating the PI3 kinase and p38 MAPK pathways," Journal of Nutritional Biochemistry, vol. 20, no. 2, pp. 106-114, 2009.

[52] R. N. DuBois, S. B. Abramson, L. Crofford et al., "Cyclooxygenase in biology and disease," FASEB Journal, vol. 12, no. 12, pp. 1063-1073, 1998.

[53] G. P. Pidgeon, J. Lysaght, S. Krishnamoorthy et al., "Lipoxygenase metabolism: roles in tumor progression and survival," Cancer and Metastasis Reviews, vol. 26, no. 3-4, pp. 503-524, 2007.

[54] Y. N. Ye, W. K. K. Wu, V. Y. Shin, I. C. Bruce, B. C. Y. Wong, and C. H. Cho, "Dual inhibition of 5-LOX and COX2 suppresses colon cancer formation promoted by cigarette smoke," Carcinogenesis, vol. 26, no. 4, pp. 827-834, 2005.

[55] B. K. Sharma, P. Pilania, and P. Singh, "Modeling of cyclooxygenase-2 and 5-lipooxygenase inhibitory activity of apoptosis-inducing agents potentially useful in prostate cancer chemotherapy: derivatives of diarylpyrazole," Journal of Enzyme Inhibition and Medicinal Chemistry, vol. 24, no. 2, pp. 607-615, 2009.

[56] M. Diederich, C. Sobolewski, C. Cerella, M. Dicato, and L. Ghibelli, "The role of cyclooxygenase-2 in cell proliferation and cell death in human malignancies," International Journal of Cell Biology, Article ID 215158, 2010.

[57] M. T. Wang, K. V. Honn, and D. Nie, "Cyclooxygenases, prostanoids, and tumor progression," Cancer and Metastasis Reviews, vol. 26, no. 3-4, pp. 525-534, 2007.

[58] D. G. Menter, R. L. Schilsky, and R. N. DuBois, "Cyclooxygenase-2 and cancer treatment: understanding the risk should be worth the reward," Clinical Cancer Research, vol. 16, no. 5, pp. 1384-1390, 2010.

[59] A. C. Reese, V. Fradet, and J. S. Witte, “ $\omega-3$ Fatty acids, genetic variants in COX-2 and prostate cancer," Journal of Nutrigenetics and Nutrigenomics, vol. 2, no. 3, pp. 149-158, 2009.

[60] H. O. Bang, J. Dyerberg, and N. Hjorne, "The composition of food consumed by Greenland Eskimos," Acta Medica Scandinavica, vol. 200, no. 1-2, pp. 69-73, 1976.

[61] J. Dyerberg, H. O. Bang, E. Stoffersen, S. Moncada, and J. R. Vane, "Eicosapentaenoic acid and prevention of thrombosis and atherosclerosis?” The Lancet, vol. 2, no. 8081, pp. 117-119, 1978. 
[62] C. N. Serhan, C. B. Clish, J. Brannon, S. P. Colgan, N. Chiang, and K. Gronert, "Novel functional sets of lipid-derived mediators with antiinflammatory actions generated from omega-3 fatty acids via cyclooxygenase 2-nonsteroidal antiinflammatory drugs and transcellular processing," Journal of Experimental Medicine, vol. 192, no. 8, pp. 1197-1204, 2000.

[63] H. Hasturk, A. Kantarci, T. Ohira et al., "RvE1 protects from local inflammation and osteoclast-mediated bone destruction in periodontitis," FASEB Journal, vol. 20, no. 2, pp. 401-403, 2006.

[64] K. M. Connor, J. P. Sangiovanni, C. Lofqvist et al., "Increased dietary intake of $\omega$-3-polyunsaturated fatty acids reduces pathological retinal angiogenesis," Nature Medicine, vol. 13, no. 7, pp. 868-873, 2007.

[65] S. Ogawa, D. Urabe, Y. Yokokura, H. Arai, M. Arita, and M. Inoue, "Total synthesis and bioactivity of resolvin E2," Organic Letters, vol. 11, no. 16, pp. 3602-3605, 2009.

[66] K. H. Weylandt, C. Y. Chiu, B. Gomolka, S. F. Waechter, and B. Wiedenmann, "Omega-3 fatty acids and their lipid mediators: towards an understanding of resolvin and protectin formation," Prostaglandins and Other Lipid Mediators, vol. 97, pp. 73-82, 2012.

[67] Y. P. Sun, S. F. Oh, J. Uddin et al., "Resolvin D1 and its aspirin-triggered 17R epimer: stereochemical assignments, antiinflammatory properties, and enzymatic inactivation," Journal of Biological Chemistry, vol. 282, no. 13, pp. 9323-9334, 2007.

[68] C. K. Park, Z. Z. Xu, T. Liu, N. Lu, C. N. Serhan et al., "Resolvin D2 is a potent endogenous inhibitor for transient receptor potential subtype V1/A1, inflammatory pain, and spinal cord synaptic plasticity in mice: distinct roles of resolvin D1, D2, and E1," Journal of Neuroscience, vol. 31, pp. 18433-18438, 2011.

[69] Y. Zhao, F. Calon, C. Julien et al., "Docosahexaenoic acidderived neuroprotectin D1 induces neuronal survival via secretase- and PPAR $\gamma$-mediated mechanisms in Alzheimer's disease models," PLoS ONE, vol. 6, no. 1, Article ID e15816, 2011.

[70] H. Tazoe, Y. Otomo, I. Kaji, R. Tanaka, S. I. Karaki, and A. Kuwahara, "Roles of short-chain fatty acids receptors, GPR41 and GPR43 on colonic functions," Journal of Physiology and Pharmacology, vol. 59, supplement 2, pp. 251-262, 2008.

[71] J. Wang, X. Wu, N. Simonavicius, H. Tian, and L. Ling, "Medium-chain fatty acids as ligands for orphan G proteincoupled receptor GPR84," Journal of Biological Chemistry, vol. 281, no. 45, pp. 34457-34464, 2006.

[72] Y. Itoh, Y. Kawamata, M. Harada et al., "Free fatty acids regulate insulin secretion from pancreatic $\beta$ cells through GPR40," Nature, vol. 422, no. 6928, pp. 173-176, 2003.

[73] A. Hirasawa, K. Tsumaya, T. Awaji et al., "Free fatty acids regulate gut incretin glucagon-like peptide-1 secretion through GPR120," Nature Medicine, vol. 11, no. 1, pp. 90-94, 2005.

[74] M. Suzuki, S. Takaishi, M. Nagasaki, Y. Onozawa, I. Iino et al., "Medium-chain fatty acid-sensing receptor, GPR84, is a proinflammatory receptor," Journal of Biological Chemistry, vol. 288, pp. 10684-10691, 2013.

[75] C. P. Briscoe, M. Tadayyon, J. L. Andrews et al., "The orphan $\mathrm{G}$ protein-coupled receptor GPR40 is activated by medium and long chain fatty acids," Journal of Biological Chemistry, vol. 278, no. 13, pp. 11303-11311, 2003.

[76] S. Hardy, G. G. St-Onge, E. Joly, Y. Langelier, and M. Prentki, "Oleate promotes the proliferation of breast cancer cells via the G protein-coupled receptor GPR40," Journal of Biological Chemistry, vol. 280, no. 14, pp. 13285-13291, 2005.
[77] T. Alquier, M. L. Peyot, M. G. Latour et al., "Deletion of GPR40 impairs glucose-induced insulin secretion in vivo in mice without affecting intracellular fuel metabolism in islets," Diabetes, vol. 58, no. 11, pp. 2607-2615, 2009.

[78] K. Nakamoto, T. Nishinaka, K. Matsumoto, F. Kasuya, M. Mankura et al., "Involvement of the long-chain fatty acid receptor GPR40 as a novel pain regulatory system," Brain Research, vol. 1432, pp. 74-83, 2012.

[79] D. Ma, M. Zhang, C. P. Larsen et al., "DHA promotes the neuronal differentiation of rat neural stem cells transfected with GPR40 gene," Brain Research, vol. 1330, pp. 1-8, 2010.

[80] A. Ichimura, A. Hirasawa, O. Poulain-Godefroy, A. Bonnefond, T. Hara et al., "Dysfunction of lipid sensor GPR120 leads to obesity in both mouse and human," Nature, vol. 483, pp. 350354, 2012.

[81] D. Y. Oh and J. M. Olefsky, "Omega 3 fatty acids and GPR120," Cell Metabolism, vol. 15, pp. 564-565, 2012.

[82] T. Kawai and S. Akira, “TLR signaling," Cell Death and Differentiation, vol. 13, no. 5, pp. 816-825, 2006.

[83] L. A. Ridnour, R. Y. Cheng, C. H. Switzer, J. L. Heinecke, S. Ambs et al., "Molecular Pathways: toll-like receptors in the tumor microenvironment: poor prognosis or new therapeutic opportunity," Clinical Cancer Research, vol. 19, no. 6, pp. 1340$1346,2012$.

[84] J. Krishnan, G. Lee, and S. Choi, "Drugs targeting toll-like receptors," Archives of Pharmacal Research, vol. 32, no. 11, pp. 1485-1502, 2009.

[85] A. Paone, D. Starace, R. Galli et al., “Toll-like receptor 3 triggers apoptosis of human prostate cancer cells through a PKC- $\alpha$ dependent mechanism," Carcinogenesis, vol. 29, no. 7, pp. 13341342, 2008.

[86] S. L. Zheng, K. Augustsson-Bälter, B. Chang et al., "Sequence variants of toll-like receptor 4 are associated with prostate cancer risk: results from the cancer prostate in Sweden study," Cancer Research, vol. 64, no. 8, pp. 2918-2922, 2004.

[87] M. R. Väisänen, T. Väisänen, A. Jukkola-Vuorinen et al., "Expression of toll-like receptor-9 is increased in poorly differentiated prostate tumors," Prostate, vol. 70, no. 8, pp. 817-824, 2010.

[88] D. Panigrahy, A. Kaipainen, E. R. Greene, and S. Huang, "Cytochrome P450-derived eicosanoids: the neglected pathway in cancer," Cancer Metastasis Reviews, vol. 29, no. 4, pp. 723-735, 2010.

[89] Z. Wang, D. Liu, F. Wang, S. Liu, S. Zhao et al., "Saturated fatty acids activate microglia via Toll-like receptor 4/NF-kappaB signalling," British Journal of Nutrition, vol. 107, pp. 229-241, 2012.

[90] G. T. Robbins and D. Nie, "PPAR gamma, bioactive lipids, and cancer progression," Frontiers in Bioscience, vol. 17, pp. 18161834, 2012.

[91] H. J. Murff, X. O. Shu, H. Li et al., "Dietary polyunsaturated fatty acids and breast cancer risk in Chinese women: a prospective cohort study," International Journal of Cancer, vol. 128, no. 6, pp. 1434-1441, 2011.

[92] H. Sun, Y. Hu, Z. Gu, R. T. Owens, Y. Q. Chen et al., “Omega3 fatty acids induce apoptosis in human breast cancer cells and mouse mammary tissue through syndecan-1 inhibition of the MEK-Erk pathway," Carcinogenesis, vol. 32, pp. 1518-1524, 2011.

[93] I. J. Edwards, I. M. Berquin, H. Sun et al., "Differential effects of delivery of omega-3 fatty acids to human cancer cells by lowdensity lipoproteins versus albumin," Clinical Cancer Research, vol. 10, no. 24, pp. 8275-8283, 2004. 
[94] H. Sun, I. M. Berquin, and I. J. Edwards, "Omega-3 polyunsaturated fatty acids regulate syndecan-1 expression in human breast cancer cells," Cancer Research, vol. 65, no. 10, pp. 4442-4447, 2005.

[95] H. Sun, Y. Hu, Z. Gu et al., "Endogenous synthesis of n-3 polyunsaturated fatty acids in Fat-1 mice is associated with increased mammary gland and liver Syndecan-1," PLoS ONE, vol. 6, no. 5, Article ID e20502, 2011.

[96] Y. H. Teng, R. S. Aquino, and P. W. Park, "Molecular functions of syndecan-1 in disease," Matrix Biology, vol. 31, pp. 3-16, 2012.

[97] T. Ishikawa and R. H. Kramer, "Sdc1 negatively modulates carcinoma cell motility and invasion," Experimental Cell Research, vol. 316, no. 6, pp. 951-965, 2010.

[98] J. Kiviniemi, M. Kallajoki, I. Kujala et al., "Altered expression of syndecan-1 in prostate cancer," APMIS, vol. 112, no. 2, pp. 89-97, 2004.

[99] D. Chen, B. Adenekan, L. Chen et al., "Syndecan-1 expression in locally invasive and metastatic prostate cancer," Urology, vol. 63, no. 2, pp. 402-407, 2004.

[100] T. Zellweger, C. Ninck, M. Mirlacher et al., “Tissue microarray analysis reveals prognostic significance of syndecan-I expression in prostate cancer," Prostate, vol. 55, no. 1, pp. 20-29, 2003.

[101] I. J. Edwards, H. Sun, Y. Hu et al., "In vivo and in vitro regulation of syndecan 1 in prostate cells by n-3 polyunsaturated fatty acids," Journal of Biological Chemistry, vol. 283, no. 26, pp. 18441-18449, 2008.

[102] P. A. Corsetto, G. Montorfano, S. Zava, I. E. Jovenitti, A. Cremona, and A. M. Rizzo, "Effects of n-3 PUFAs on breast cancer cells through their incorporation in plasma membrane," Lipids in Health and Disease, vol. 10, article 73, 2011.

[103] J. B. Ewaschuk, M. Newell, and C. J. Field, "Docosahexanoic acid improves chemotherapy efficacy by inducing CD95 translocation to lipid rafts in ER(-) breast cancer cells," Lipids, vol. 47, pp. 1019-1030, 2012.

[104] J. Y. Lee, L. Zhao, and D. H. Hwang, "Modulation of pattern recognition receptor-mediated inflammation and risk of chronic diseases by dietary fatty acids," Nutrition Reviews, vol. 68, no. 1, pp. 38-61, 2010.

[105] Y. Mitsuishi, H. Motohashi, and M. Yamamoto, "The Keap1-Nrf2 system in cancers: stress response and anabolic metabolism," Frontiers in Oncology, vol. 2, article 200, 2012.

[106] H. Wang, T. O. Khor, C. L. L. Saw et al., "Role of Nrf2 in suppressing LPS-induced inflammation in mouse peritoneal macrophages by polyunsaturated fatty acids docosahexaenoic acid and eicosapentaenoic acid," Molecular Pharmaceutics, vol. 7, no. 6, pp. 2185-2193, 2010.

[107] P. Zhang, A. Singh, S. Yegnasubramanian et al., "Loss of kelch-like ECH-associated protein 1 function in prostate cancer cells causes chemoresistance and radioresistance and promotes tumor growth," Molecular Cancer Therapeutics, vol. 9, no. 2, pp. 336-346, 2010.

[108] A. Singh, S. Boldin-Adamsky, R. K. Thimmulappa et al., "RNAimediated silencing of nuclear factor erythroid-2-related factor 2 gene expression in non-small cell lung cancer inhibits tumor growth and increases efficacy of chemotherapy," Cancer Research, vol. 68, no. 19, pp. 7975-7984, 2008.

[109] L. Gao, J. Wang, K. R. Sekhar et al., "Novel n-3 fatty acid oxidation products activate Nrf2 by destabilizing the association between Keap1 and Cullin3," Journal of Biological Chemistry, vol. 282, no. 4, pp. 2529-2537, 2007.
[110] A. Ishikado, Y. Nishio, K. Morino et al., "Low concentration of 4-hydroxy hexenal increases heme oxygenase-1 expression through activation of $\mathrm{Nrf} 2$ and antioxidative activity in vascular endothelial cells," Biochemical and Biophysical Research Communications, vol. 402, no. 1, pp. 99-104, 2010.

[111] H. Wang, T. O. Khor, C. L. L. Saw et al., "Role of Nrf2 in suppressing LPS-induced inflammation in mouse peritoneal macrophages by polyunsaturated fatty acids docosahexaenoic acid and eicosapentaenoic acid," Molecular Pharmaceutics, vol. 7, no. 6, pp. 2185-2193, 2010.

[112] M. J. Magbanua, R. Roy, E. V. Sosa, V. Weinberg, S. Federman et al., "Gene expression and biological pathways in tissue of men with prostate cancer in a randomized clinical trial of lycopene and fish oil supplementation," PLoS One, vol. 6, no. 9, Article ID e24004, 2011.

[113] B. Minke, "TRP channels and $\mathrm{Ca}^{2+}$ signaling," Cell Calcium, vol. 40, no. 3, pp. 261-275, 2006.

[114] J. A. Matta, R. L. Miyares, and G. P. Ahern, "TRPV1 is a novel target for omega-3 polyunsaturated fatty acids," Journal of Physiology, vol. 578, no. 2, pp. 397-411, 2007.

[115] M. Parnas, M. Peters, and B. Minke, "Linoleic acid inhibits TRP channels with intrinsic voltage sensitivity: implications on the mechanism of linoleic acid action," Channels, vol. 3, no. 3, pp. 164-166, 2009.

[116] M. Leonelli, M. F. Graciano, and L. R. Britto, “TRP channels, omega- 3 fatty acids, and oxidative stress in neurodegeneration: from the cell membrane to intracellular cross-links," Brazilian Journal of Medical and Biological Research, vol. 44, pp. 10881096, 2011.

[117] S. Ye, L. Tan, J. Ma, Q. Shi, and J. Li, "Polyunsaturated docosahexaenoic acid suppresses oxidative stress induced endothelial cell calcium influx by altering lipid composition in membrane caveolar rafts," Prostaglandins Leukotrienes and Essential Fatty Acids, vol. 83, no. 1, pp. 37-43, 2010.

[118] M. Flourakis and N. Prevarskaya, "Insights into $\mathrm{Ca}^{2+}$ homeostasis of advanced prostate cancer cells," Biochimica et Biophysica Acta, vol. 1793, no. 6, pp. 1105-1109, 2009.

[119] Y. Sun, S. Selvaraj, A. Varma, S. Derry, A. E. Sahmoun et al., "Increase in serum $\mathrm{Ca}^{2+} / \mathrm{Mg}^{2+}$ ratio promotes proliferation of prostate cancer cells by activating TRPM7 channels," Journal of Biological Chemistry, vol. 288, pp. 255-263, 2013. 

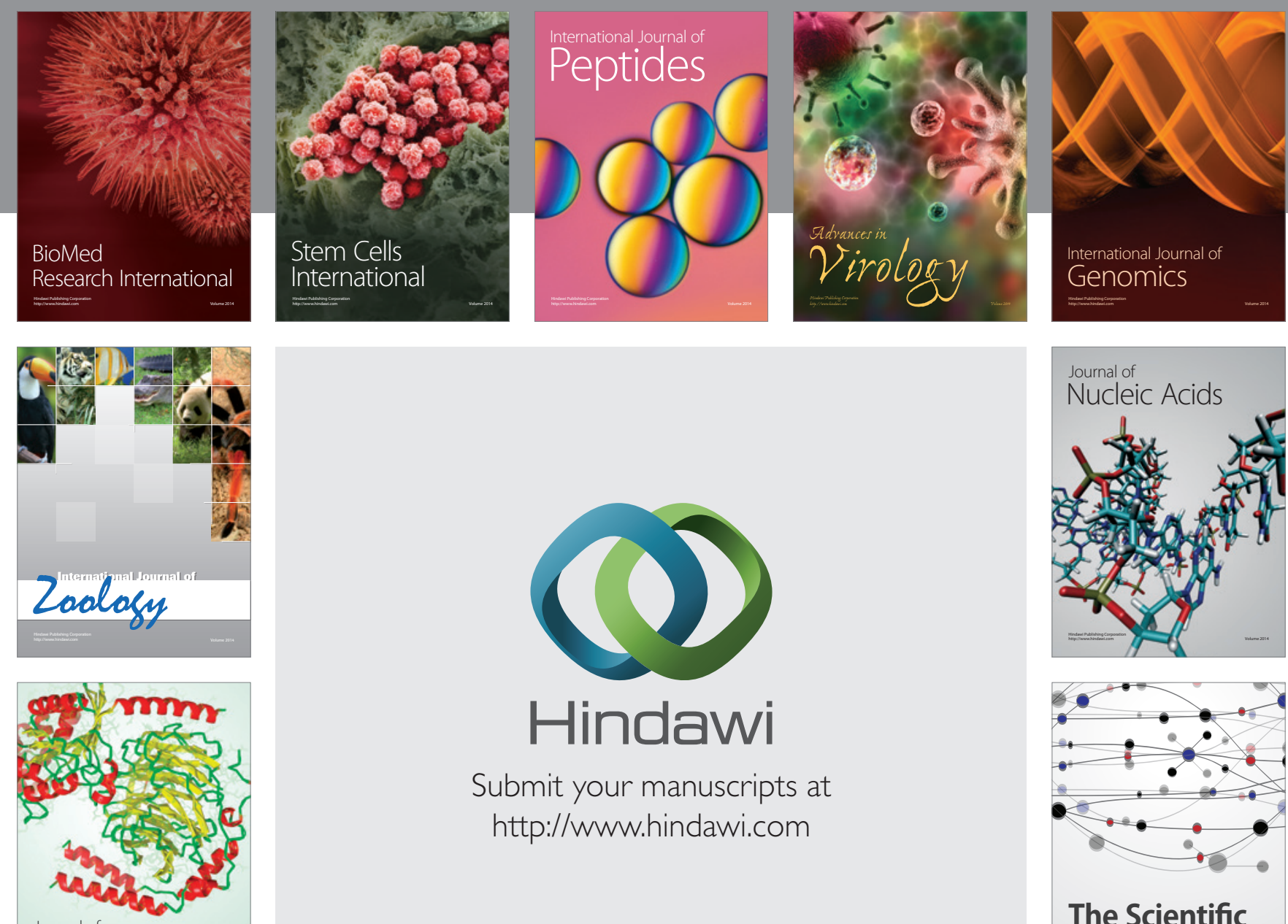

Submit your manuscripts at

http://www.hindawi.com

Journal of
Signal Transduction
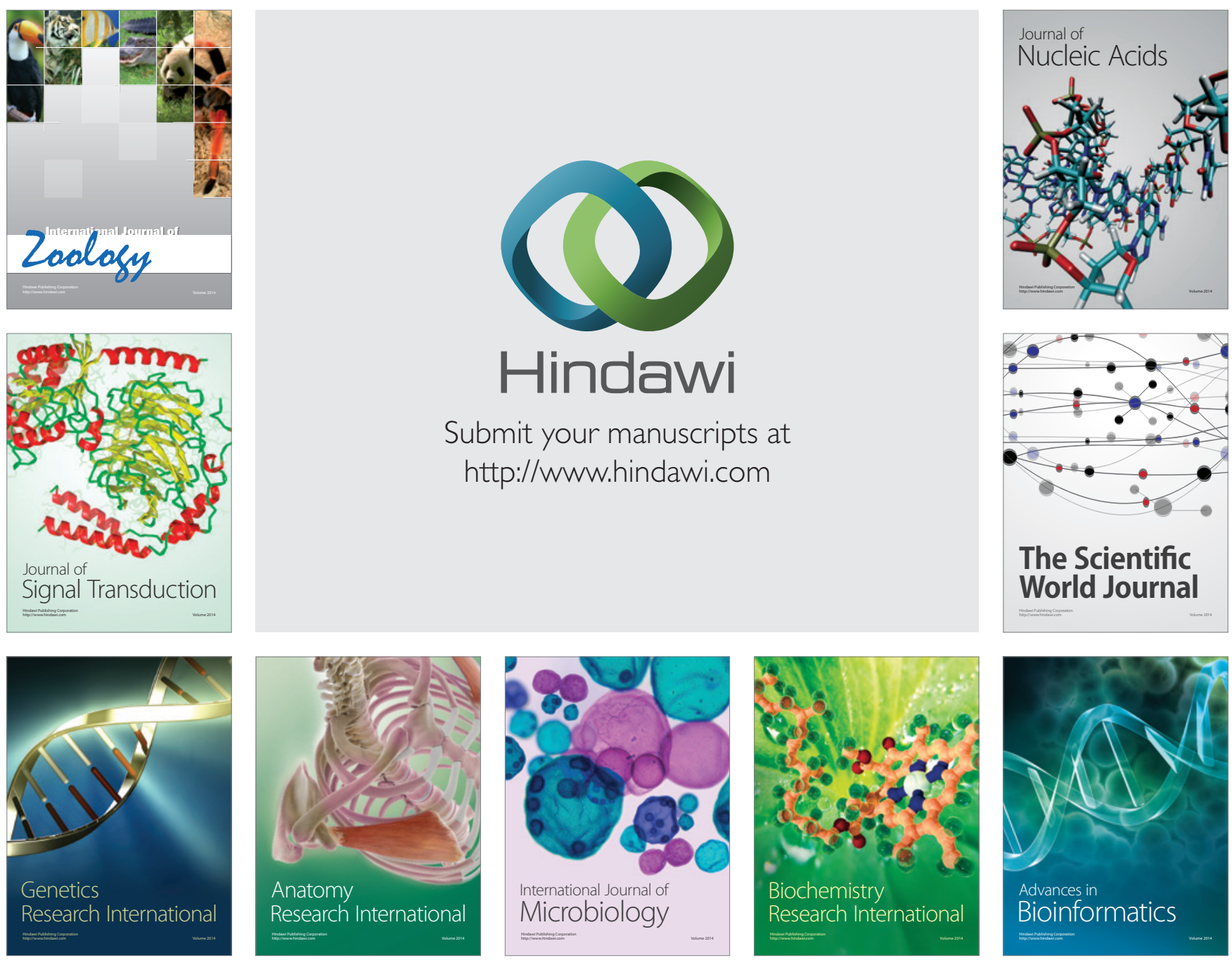

The Scientific World Journal
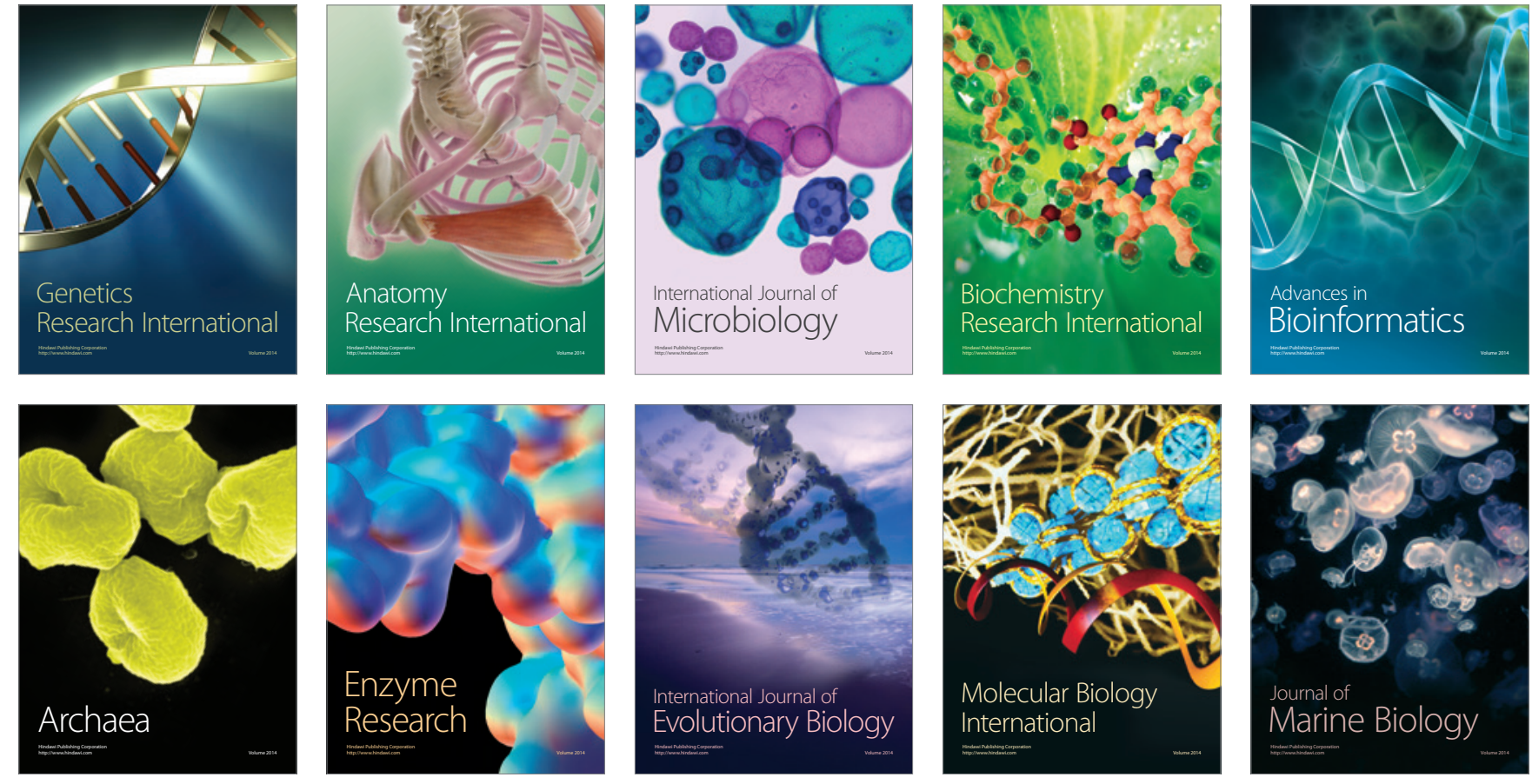